\title{
XXXIVth Seminar of the French-Speaking Society for Theoretical Biology: Saint-Flour (Cantal), France, 26-28 May, 2014
}

\author{
Nicolas Glade ${ }^{1}$ Angélique Stéphanou ${ }^{1}$
}

Received: 17 July 2015/ Accepted: 20 July 2015/Published online: 28 July 2015

(C) Springer Science+Business Media Dordrecht 2015

Historically, theoretical biology was built on mathematics in Europe. The FrenchSpeaking Society of Theoretical Biology (SFBT), strong with a long tradition in biomathematics, federates theoretical biology in French-Speaking countries and France in particular. For several years now, with the increase of computational means and the development of research in the domain of complex systems, the framework of theoretical biology evolved towards more computational approaches. The Spring School 2012 of the SFBT reflected this trend by addressing the theme of numerical experimentations and hybrid approaches which associate continuous and discrete models. On this 2014 edition of the seminar of the society, we wished to go one step further by promoting discussions on the subject of Artificial Life.

Both Theoretical Biology and Artificial Life aim to understand the Living. The difference is essentially a semantical one and is often linked to the scale at which the problem is addressed. In theoretical biology, we "make models", often along a topdown approach on the basis of mathematical equations. In artificial life, we "simulate virtual words" along a reciprocal bottom-up approach based on numerical entities. As different as they may appear and as different as the associated communities may be, their aim remains the same: deciphering the mechanisms of the Living and forecasting its evolution. The two approaches converge in the sense that each provides some elements of understanding. The first paper by Atangana "Modeling the enzyme kinetic reaction" is such a contribution for the understanding of enzymatic mechanisms. In the other hand, the following paper by Bedessem

Angélique Stéphanou

angelique.stephanou@imag.fr

Nicolas Glade

nicolas.glade@imag.fr

1 UJF-Grenoble 1, CNRS, Laboratory TIMC-IMAG/DyCTiM, 38041 Grenoble, France 
and Ruphy "SMT or TOFT? How the two main theories of carcinogenesis are made (artificially) incompatible" is a philosophically grounded investigation to analyze and understand the (apparent) opposition between two conceptual models of cancer.

Once understood, the Living can be controlled to become an exploitable device for engineering. Its counterpart, the model (or the virtual word) is the theoretical tool that allows to design and test such physical devices. For example, the design of an artifical kidney can be made possible by a functional model of the organ. A directly related contribution is presented by Fontecave-Jallon and Thomas "Implementation of a model of bodily fluids regulation". The control of the regeneration of organisms as presented by Tosenberger et al. in "A conceptual model of morphogenesis and regeneration" is another example, but at the cell level.

At this stage of the understanding of the Living and of its control, it is conceivable to hijack it from its primary function to produce programmable biological systems inspired from the architecture and the functioning of electronic machines. Consequently, the emerging and finely controlled morphogenetical process, allows the making of "organisms-machines" as proposed by Varenne et al. in "Programming the emergence in morphogenetically architected complex systems". Beyond this, synthetic biology allows one to hijack the classical use of the elements of the Living such as vesicles or macromolecules to propose new computing devices exploiting the unrivalled characteristics of the Living in terms of parallelism, robustness, resilience for which two illustrations are given in the contributions of Courbet et al. "Computing with synthetic protocells" and BassoBlandin and Delaplace "Towards a behavioral-matching based compilation of synthetic biology functions".

We thank the authors of the seven papers that compose this special issue which contibutes to show the diversity and ongoing evolution of theoretical biology.

The SFBT wishes to pay tribute to Philippe Tracqui who for long has contributed to theoretical biology in his research works, in particular in the domains of mechanobiology and in the understanding of morphogenesis and also within the SFBT in which he was for many years very much involved.

Acknowledgments We wish to thank Dusan Misevic for introducting the 34th seminar of the SFBT with his presentation on Artificial Life entitled "Sex and cooperation in digital communities". We are also very greatful to our sponsors for the organization of this event: GDR Phenix, GDR METICE, and the AIM (Association d'Informatique Médicale). 